\title{
VERZEICHNIS DER ABKÜRZUNGEN, SIGLEN UND ZEICHEN
}

Die Siglen, die in den Apparaten (vor allem im apparatus fontium) verwendet werden, werden direkt vor der Edition nachgewiesen. Für Literaturverweise insgesamt sei auf das Literaturverzeichnis verwiesen. Hier werden auch Abkürzungen entschlüsselt, deren Verständnis an sich einfach ist.

Abkürzungen und Zeichen im gesamten Text

$\begin{array}{ll}\text { App. } & \text { Apparat, apparatus } \\ \text { armen. } & \text { armenisch, armeniace } \\ \text { bes. } & \text { besonders, praecipue } \\ \text { bzw. } & \text { beziehungsweise, respective } \\ \text { cit. } & \text { von Or., pasch. ed. Guéraud/Nautin S. 63-75 als „,itatio“ } \\ & \text { angeführt, citatio (Or., pasch. ed. Guéraud/Nautin S. 63-75) } \\ \text { ed. } & \text { edidit / ediderunt } \\ \text { Einl. } & \text { Einleitung, introductio } \\ \text { Etym. } & \text { Etymologie, etymologia } \\ \text { f. } & \text { und folgende(r), et sequens } \\ \text { ff. } & \text { und folgende, et sequentes } \\ \text { Forts. } & \text { Fortsetzung, continuatio } \\ \text { lat. } & \text { lateinisch, latine } \\ \text { s. } & \text { siehe, vide } \\ \text { S. } & \text { Seite(n),pagina(e) } \\ \text { s. o. } & \text { siehe oben, vide supra } \\ \text { s. u. } & \text { siehe unten, vide infra } \\ \text { t.r. } & \text { textus receptus } \\ \text { Testim. } & \text { Testimonium, testimonium } \\ \text { u. ö. } & \text { und öfter, et alibi } \\ \text { Übers. } & \text { Übersetzung, translatio } \\ \text { übers. } & \text { übersetzt von, transtulit, transtulerunt } \\ \text { verm. } & \text { vermutlich, probabiliter } \\ \text { viell. } & \text { vielleicht, fortasse } \\ \text { vgl. } & \text { vergleiche, confer } \\ \text { z. B. } & \text { zum Beispiel, exempli gratia } \\ \text { z. St. } & \text { zur Stelle, ad locum } \\ \text { Zuschr. } & \text { Zuschreibung, nominatio auctoris } \\ & \end{array}$


Biblische Bücher

Biblische Bücher werden nach den Richtlinien der Editionsreihe so abgekürzt:

\begin{tabular}{|c|c|}
\hline Gen & Genesis \\
\hline Ex & Exodus \\
\hline Lev & Leviticus \\
\hline Num & Numeri \\
\hline Dtn & Deuteronomium \\
\hline Ios & Josua \\
\hline $\mathrm{Idc}$ & Judicum \\
\hline I. / II. Sam & I. / II. Samuelis $\left(B \propto \sigma \iota \lambda \varepsilon \mid \tilde{\omega} \nu A^{\prime} / B^{\prime}\right)$ \\
\hline I. / II. Reg & 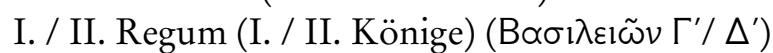 \\
\hline I. / II. Chron & I. / II. Chronicorum \\
\hline Tob & Tobias \\
\hline I. / IV. Macc & I. / IV. Makkabäer \\
\hline Ps & Psalmen \\
\hline Od & Oden \\
\hline Prov & Proverbia (Sprüche Salomonis) \\
\hline Cant & Canticum Canticorum (Hohes Lied) \\
\hline $\mathrm{Hi}$ & Hiob \\
\hline Sap & Sapientia Salomonis \\
\hline Sir & Jesus Sirach \\
\hline Hos & Hosea \\
\hline Am & Amos \\
\hline $\mathrm{Mi}$ & Micha \\
\hline Ioel & Joel \\
\hline $\mathrm{Ob}$ & Obadja (Abdias) \\
\hline Io & Jona \\
\hline Nah & Nahum \\
\hline $\mathrm{Hab}$ & Habakuk \\
\hline Hag & Haggai \\
\hline Sach & Sacharia \\
\hline Mal & Maleachi \\
\hline Ies & Jesaia \\
\hline Ier & Jeremia \\
\hline Bar & Baruch \\
\hline Lam & Lamentationes (Klagelieder Jeremiae) \\
\hline Hes & Hesekiel (Ezechiel) \\
\hline Dan & Daniel \\
\hline
\end{tabular}




$\begin{array}{ll}\text { Mt } & \text { Matthäus } \\ \text { Mc } & \text { Marcus } \\ \text { Lc } & \text { Lucas } \\ \text { Ioh } & \text { Johannes } \\ \text { Act } & \text { Acta Apostolorum (Apostelgeschichte) } \\ \text { Rm } & \text { Römerbrief } \\ \text { I. / II. Cor } & \text { I. / II. Korintherbrief } \\ \text { Gal } & \text { Galaterbrief } \\ \text { Eph } & \text { Epheserbrief } \\ \text { Phil } & \text { Philipperbrief } \\ \text { Col } & \text { Kolosserbrief } \\ \text { I. / II. Thess } & \text { I. / II. Thessalonicherbrief } \\ \text { I. / II. Tim } & \text { I. / II. Timotheusbrief } \\ \text { Tit } & \text { Titusbrief } \\ \text { Hebr } & \text { Hebräerbrief } \\ \text { Iac } & \text { Jakobusbrief } \\ \text { I. / II. Ptr } & \text { I. / II. Petrusbrief } \\ \text { I. / II. Ioh } & \text { I. / II. Johannesbrief } \\ \text { Iud } & \text { Judasbrief } \\ \text { Apc } & \text { Apokalypse (Offenbarung) } \\ & \\ & \end{array}$

[R] Lemma, das bei Prokop nicht an der Stelle steht, wo es nach biblischer Reihenfolge stehen müsste, locus biblicus ordine discrepans ab ordine biblico

\section{Zeichen im textus receptus}

xxx nicht lesbare Buchstaben (überschrieben oder ausradiert), litterae hand legibiles

$\dagger \ldots \dagger \quad$ Korruptele, textus corruptus

() Parenthese (Einschaltung des antiken Autors, nach Gestaltung der Herausgeberin), parenthesis auctoris durch Konjektur gewonnener Zusatz, additio per coniecturam Wechsel der Spalten in der Migneedition (PG 87/1), die am inneren Rand der Kolumnenzeile aufgeschlüsselt werden, mutatio columnarum in editione PG 87/1 


\section{Zitatkennzeichnungen im textus receptus}

» Zitat aus dem an dieser Stelle kommentierten Bibeltext (Lem$\mathrm{ma})$, citatio lemmatis

Kursivschreibung, litterae cursivae

Sonstige Zitate, z. B. Neues Testament, übrige Septuaginta; sonstige griechische Übersetzer des Alten Testaments; nichtbiblische Zitate, citatio ex aliis textibus

' fiktives Zitat, ${ }^{340}$ citatio non specifica

\section{Abkürzungen und Zeichen im apparatus criticus}

]

$+$

$<$

$\sim$

a. Rd.

dopp.

erg.

fen.

Hss.

konj.

korr.

n. lesb.

ü. d. Z.

Zch.

$\mathrm{Zl}$.
Abtrennung zwischen Lemma und (1.) abweichender Lesart divisio lemmatis et lectionis codicis / lectionum codicum

hat / haben hinzugefügt, addidit / addiderunt

hat / haben ausgelassen, omisit / omiserunt

hat / haben umgestellt, transposuit / transposuerunt

am Rand, in margine

hat / haben verdoppelt, duplicavit / duplicaverunt

hat ergänzt, addidit

Fenestra (in der Handschrift frei gelassener Platz), exbibet fenestram

Verschiedene Zustände einer Handschrift werden so unterschieden:

A* Zustand vor Korrektur, ante correcturam

340 Mit diesen Anführungszeichen werden verschiedene Zitatarten gekennzeichnet, darunter ein fingierter Wortlaut, der sich gerade nicht in der Bibel findet, ein Begriff, eine Übersetzung (z. B. eines griechischen Begriffes oder eines hebräischen oder syrischen Wortes oder Satzes); eine Umschrift (z. B. des hebräischen Wortlauts); eine etymologische oder biblische Deutung eines nichtgriechischen Wortes (insbesondere Namens) oder die Anspielung auf eine solche Deutung (z. B. ' $\delta 1 \varepsilon$ -

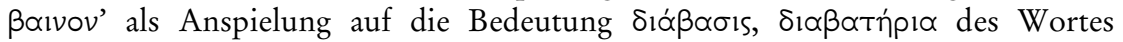

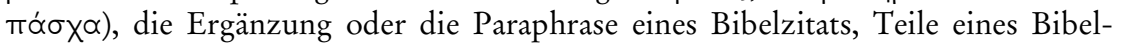
zitats, die ergänzt oder nicht wörtlich zitiert werden. 
$\mathrm{A}^{\mathrm{I}} \quad$ Korrektur von der Hand des ersten Schreibers, correctura prima manu

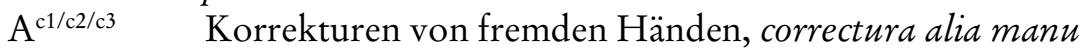

$\mathrm{A}^{\mathrm{c}} \quad$ keinem bestimmten Schreiber oder Korrektor zuordenbare Korrekturen, correctura incerta manu

\section{Abkürzungen im apparatus fontium}

Aqu. Aquila

Ged. auch / zum Ged. vgl.

Gedanke auch / zum Gedanken vergleiche / verwandter Gedanke (entfernte Parallele, bei denen nicht sicher ist, ob Prokop oder der Kompilator der Urkantene das genannte Werk gekannt hat), confer latu sensu

Gedanke auch / zum Gedanken vergleiche / verwandter Gedanke

(entfernte Parallele, bei denen die Kenntnis des Werks oder seiner Quelle bei Prokop oder der Urkatene nicht sicher ist), confer latu sensu

Hebr. $\quad$ Übersetzung des hebräischen Textes, translatio textus hebraici

hexapl. hexaplarische Lesart, nicht näher bestimmte jüngere Übersetzung des Bibeltextes, incerta recentior translatio textus veteris testamenti

LXX Septuaginta

nicht identif. Fragment, dessen Herkunft mit den benutzten Hilfsmitteln nicht bestimmt werden konnte, fontem non inveni

Symm. Symmachus

Syr. $\quad$ syrische Übersetzung, translatio Syriaca

Theod. Theodotion

vgl. vergleiche (nichtwörtliche Anführung, ein im Wortlaut verändertes Zitat oder eine Gedankenparallele, für die ein direkter Bezug Prokops bzw. der Urkatene angenommen werden kann, sei es ein Bezug zu dem genannten Werk oder zu dessen Quelle), confer 